\title{
Upper gastrointestinal bleeding in chronic pancreatitis
}

\author{
B. P. Kandel, B. Ghimire, P. J. Lakhey, U. K. Shrestha, M. Khakurel \\ Department of Surgery, Tribhuvan University Teaching Hospital, Kathmandu, Nepal \\ Correspondence to: Dr. Bishnu Prasad Kandel, Department of Surgery, Tribhuvan University Teaching Hospital, Kathmandu, \\ Nepal \\ Email: drkandel@hotmail.com
}

\begin{abstract}
Pseudoaneurysm of peripancreatic vessels is a rare complication of chronic pancreatitis. Being close to the pancreas, splenic artery is the most frequently affected vessel whereas right gastroepiploic artery pseudoaneurysm is very rare. Rupture of such pseudoaneurysm is a rare cause of upper gastrointestinal (GI) bleeding. We report a patient of chronic pancreatitis with past history of pancreatic duct stenting who presented with massive upper GI bleeding and stent migration. Bleeding from pseudoaneurysm of right gastroepiploic artery was detected in angiography. Coil embolization was done but later rebleeding occurred and was managed with laparotomy and ligation of the pseudoaneurysm. Patient improved during postoperative period.
\end{abstract}

Keywords: chronic pancreatitis, embolization, pseudoaneurysm

\section{Introduction}

Splanchnic arterial pseudoaneurysm is a rare complication of chronic pancreatitis. Unruptured pseudoaneurysms are usually asymptomatic. They can rupture into the gastrointestinal tract, peritoneal cavity, retroperitoneum or pancreatic duct causing the most rapidly fatal complication of chronic pancreatitis. Most frequently the pseudoaneurysm ruptures and bleeds into the gastrointestinal tract. ${ }^{1,2}$ Ten percent of chronic pancreatitis are associated with pseudoaneurysm of peripancreatic arteries and rupture occurs in 50\% of them. ${ }^{3-5}$ It can involve vessels including splenic artery, gastroduodenal artery, gastric artery, gastroepiploic artery or hepatic artery but splenic artery is commonly affected ${ }^{6}$ Clinical diagnosis is very difficult before rupture occurs but it can be detected by doppler ultrasound. We report a case of chronic pancreatitis complicated with bleeding from right gastroepiploic artery pseudoaneurysm.

\section{Case report}

A 14-year boy presented with massive hematemesis and melena for two days. He was being treated for chronic pancreatitis and was in regular follow up in other center. He had undergone endoscopic retrograde cholangiopancreatography (ERCP) and pancreatic duct stenting three months back. Clinical examination findings were noncontributory except that the patient was pale. Initial hemoglobin was $5.6 \mathrm{gm} / \mathrm{dl}$, pancreatic enzymes and liver function tests were within normal limits. $\mathrm{X}$ ray of abdomen showed calcifications in upper abdomen and stent on right iliac fossa (Fig. 1). Ultrasound (USG) abdomen with doppler was suggestive of bleeding from pseudoaneurysm. Total of seven units of blood was transfused during resuscitation. Conventional angiography confirmed the diagnosis of pseudoaneurysm of right gastroepiploic artery and coil embolization of the aneurysm was done. The patient improved after the procedure. After seven days he again developed massive upper GI bleeding. Doppler USG revealed the pseudoaneurysm fed by left gastroepiploic artery. After resuscitation, the patient underwent exploratory laparotomy. Pancreas was adherent to stomach and the pseudoaneurysm could be felt behind the antrum of stomach. Through trans-gastric approach, the aneurysm was accessed and plicated. The migrated stent had reached the terminal ileum and was removed by enterotomy. Postoperative recovery was uneventful and he is asymptomatic till one year follow up after surgery. 


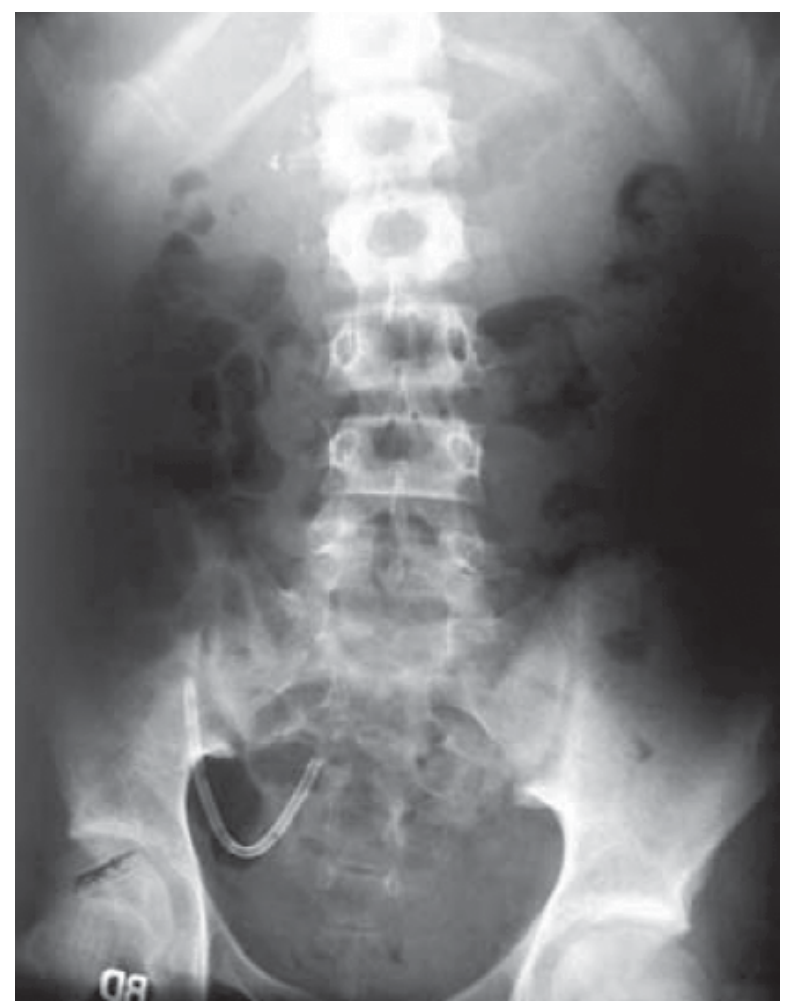

Fig. 1: Abdomen $\mathrm{X}$ ray showing calcification on pancreas and stent in right iliac fossa

\section{Discussion}

A visceral pseudoaneurysm is a serious complication of pancreatitis. Location of pseudoaneurysm depends on the distribution of fluid collection and pseudocyst in pancreatitis. Most commonly involved arteries are splenic, gastroduodenal, and pancreaticoduodenal, which account for up to $90 \%$ of total pseudoaneurysm. ${ }^{7}$ Gastroepiploic artery pseudoaneurysm is very rare. Two different mechanisms are proposed for the development of pseudoaneurysm. It can develop either due to autodigestion of the arterial wall by proteolytic enzymes or by erosion of a pseudocyst into the artery and conversion of its cavity into a pseudoaneurysm. ${ }^{5,7}$ Pseudoaneurysms can rupture into the gastrointestinal tract, peritoneal cavity, retroperitoneum, biliopancreatic ducts or pseudocysts. ${ }^{5,8}$ Factors that increase risk of bleeding in chronic pancreatitis includes duration of disease; proximity of a vessel to a pseudocyst; communication with the bile or pancreatic duct and splenic vein occlusion from thrombosis. ${ }^{3}$ None of these risk factors were present in our patient.

A bleeding pseudoaneurysm may manifest as gradually increasing anemia with melena or as intermittent massive bleeding into the gastrointestinal tract or peritoneal cavity., ${ }^{9}$ Rupture into a pseudocyst or into peritoneal cavity causes acute abdominal pain. Signs and symptoms are non specific and clinical diagnosis of ruptured pseudoaneurysm is rarely possible. Pseudoaneurysm rupture is not a common cause of GI bleeding even in patients with chronic pancreatitis. Our patient presented with features of massive upper GI bleeding. Early diagnosis and appropriate treatment of this rare complication is extremely important as it has a reported mortality rate of up to $29 \%$ with treatment and up to $90 \%$ without treatment. ${ }^{7}$ USG abdomen is the first line investigation but the findings are usually nonspecific. Doppler USG or computed tomography can help in the diagnosis. Angiography is the gold standard procedure for imaging pseudoaneurysms. ${ }^{8}$ Angiography is required for treatment with embolization or localizing the lesion if surgery is planned. In our patient the pseudoaneurysm was suggested by Doppler USG and conformed by conventional angiography (Fig. 2).

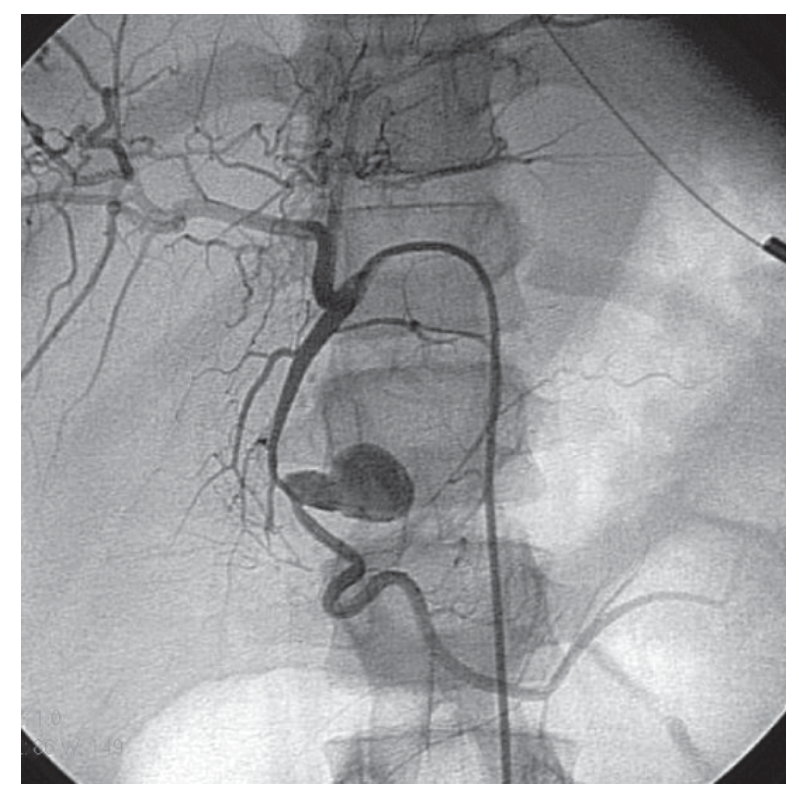

Fig. 2: Angiography showing aneurysm of right gastroepiploic artery

Optimal treatment of bleeding pseudoaneurysm remains controversial. ${ }^{9}$ No evidence-based guidelines exist regarding the treatment modality as limited data is available. Treatment options include angiographic embolization and surgical ligation of the pseudoaneurysm with or without pancreatic resection. Aneurysm trapping by embolizing proximal and distal to the aneurysm is recommended for 
successful outcome. In our patient transfemoral visceral angiography and coil embolization was performed by experienced radiological interventionalists and good hemostasis was achieved (Fig. 3).

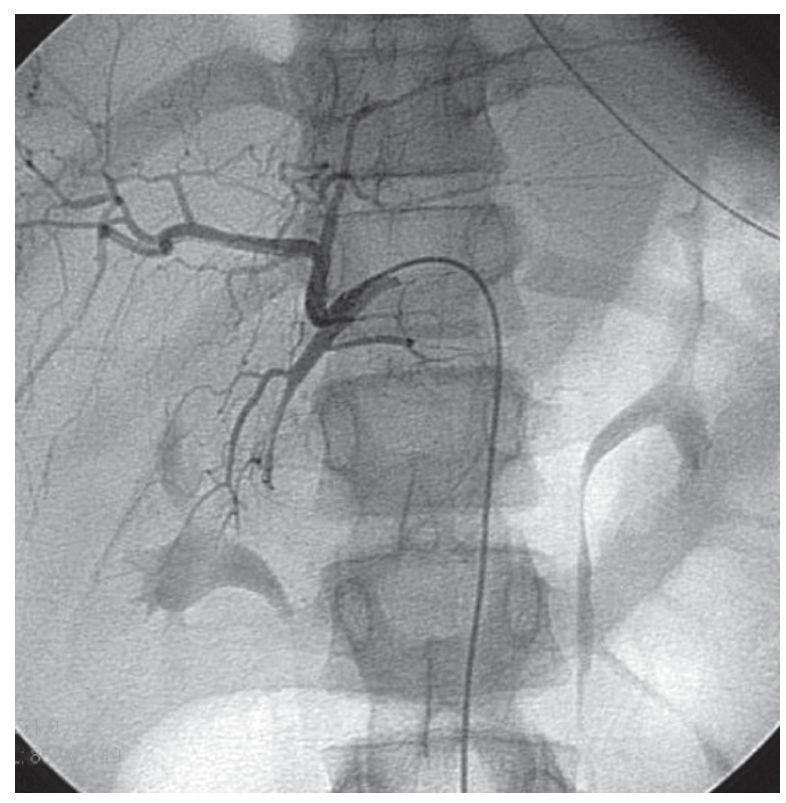

Fig. 3: Angiography after coil embolization of the aneurysm

Some authors suggest initial embolization of pseudoaneurysms as the treatment of choice with subsequent surgical intervention in case of recurrent hemorrhage. 7,9 The reported success of embolization is 60 $100 \%$ and mortality rate after embolization is $12-33 \%{ }^{10,11}$ Controversy also exists about the definition of re-bleeding after embolization. Some authors define it as rebleeding within 48 hours after embolization and their result shows high success rates. In some literature it is defined as rebleeding within one month and their success rate of embolization is $20 \% .^{7,10}$ Rebleeding occurred in our patient after one week and the aneurysm was found to be fed by left gastroepiploic artery.

Embolization fails to address the underlying disease and subsequent surgery is usually indicated. ${ }^{1,8}$ Surgical procedures can be pseudoaneurysm excision and arteriorrhaphy, total or partial pancreatectomy, ligation of bleeder and pseudocyst drainage. Some researchers suggest that proximal and transcystic ligation of a bleeding vessel is superior to pancreatic resection. ${ }^{4}$ Proponents of pancreatic resection suggest that it is the only way to prevent rebleeding so it should be employed liberally. ${ }^{10}$ But, pancreatic resection has its own morbidity. Laparotomy was done in our patient as he developed rebleeding.
Intraoperative bleeding was not extensive which could be attributed to previous embolization of the major feeding vessel (Fig. 4). Pancreatic resection was not done as it would be a major surgical procedure around a dense adhesion in a young unstable patient. The surgical treatment of the patients should be individualized. Location of a pseudoaneurysm is a major issue when selecting surgical options. Distal pancreatectomy and splenectomy should be employed to treat bleeding lesions located in the pancreatic tail as these procedures have low morbidity and mortality. ${ }^{10}$ Pancreaticoduodenectomy should be limited to selected situations for which less invasive procedures are not technically feasible. ${ }^{11,12}$ Favorable result has been reported in two-thirds of patients when the operative strategy was control of bleeding and removal of the affected pancreatic segment. ${ }^{4}$ Reported success rate of surgical intervention is up to $89 \%{ }^{3}$

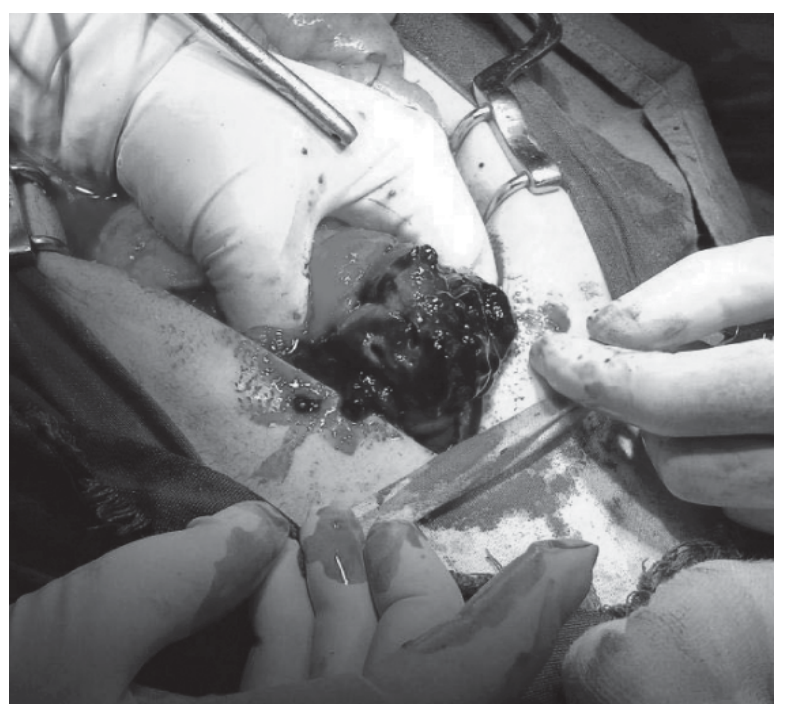

Fig. 4: Blood clot in the aneurysm

\section{Conclusions}

Rupture of pseudoaneurysm of peripancreatic vessels can present as upper GI bleeding and has a high mortality rate. Angiography is the gold standard for imaging pseudoaneurysm and embolization of the bleeding vessel can be a life saving procedure. At times they need surgical ligation of the bleeder with or without resection of affected pancreas.

\section{References}

1 Hourani SA, Al-Bdour MN, Rashaideh MA, AlNawayseh KR, Alasmar AA, Alkasasbeh MA, et al. 
Pseudo aneurysm complicates pancreatic pseudo cyst: importance of early detection and management. J Surg Pak. 2008;13:42-5.

2 Bresler L, Boissel P, Grosdidier J. Major hemorrhage from pseudocysts and pseudoaneurysms caused chronic pancreatitis: surgical therapy. World J Surg. 1991;15:649-53.

3 Hsu JT, Yeh CN, Hung CF, Chen HM, Hwang TL, Jan YY, et al. Management and outcome of bleeding pseudoaneurysm associated with chronic pancreatitis. BMC Gastroenterol. 2006;6.

4 El Hamel A, Parc R, Adda G, Bouteloup PY, Huguet C, Malafosse M. Bleeding pseudocysts and pseudoaneurysms in chronic pancreatitis. Br J Surg. 1991;78:1059-63.

5 Lee HS, park JJ, kim CD, Ryu HS, Hynu JH. Pseudoaneurysm and splenic infraction in chronic pancreatitis. J Kor Med Sces. 1996;11:183-7.

6 Benoit L, Fraisse J, Cercueil JP, Cornet A, Cuisenier J. Gastroduodenal arterial aneurysm and chronic pancreatitis. A case and review of the literature. Ann Chir. 1996;50:918-20.

7 Johnson C, Chahin C, Rose B, Goyal M. Ruptured intracystic pseudoaneurysm of the left gastric artery, which complicated pancreatitis. Applied Radiology. 2008;37.

8 Tessier DJ. Clinical features and management of splenic artery pseudo aneurysm: case series and cumulative review of literature. J Vasc Surg. 2003;38:969-74.

9 Bergert H, Hinterseher I, Kersting S, Leonhardt J, Bloomenthal A, Saeger HD. Management and outcome of hemorrhage due to arterial pseudoaneurysms in pancreatitis. Surg. 2005;137: 323-8.

10 de Perrot M, Berney T, Buhler L, Delgadillo X, Mentha G, Morel P. Management of bleeding pseudoaneurysms in patients with pancreatitis. Br J Surg. 1999;86:29-32.

11 Gould JE. Angiography and embolization in lower gastrointestinal bleeding. Applied Radiology. 2004;33.

12 Pitkaranta P, Haapiainen R, Kivisaari L, Schroder T. Diagnostic evaluation and aggressive surgical approach in bleeding pseudoaneurysms associated with pancreatic pseudocysts. Scand J Gastroenterol. 1991;26:58-64. 